\title{
Automated Preoperative Planning of Acetabulam Size and Angle Detection for Hemi-arthroplasty
}

\author{
Himanka Sekhar Mondal \\ Central Scientific Instruments \\ Organisation \\ Chandigarh, India
}

\author{
Shashi Sharma \\ Central Scientific Instruments \\ Organisation \\ Chandigarh, India
}

\author{
Amod Kumar \\ Central Scientific Instruments \\ Organisation \\ Chandigarh India
}

\begin{abstract}
Conventional pre-operative planning for Hemi-arthroplasty is performed with caliper, protractor, transparent templates and $\mathrm{x}$-ray sheets. This technique is time consuming with many errors. Computer-aided pre-operative planning systems can assist surgeons in selecting correct sized implantable acetabulam and accurate planning of an operation. This paper describes the pre-operative planning by digital image processing techniques for Total Hip Replacement by using 2D digital X-ray images which leads to the development of a software for surgeons and radiographers. The authors have used advanced image processing techniques and algorithms in image enhancement, calibration, planning, templating and reporting to overcome problems faced while doing all these manually. The proposed methodology provides accurate, computer programmed, user-friendly and dimensionally correct solution. The technique seems to be reliable and acceptable to patients, radiographers and surgeons
\end{abstract}

\section{Keywords}

Total Hip Replacement, Preoperative Planning, Digital Image processing

\section{INTRODUCTION}

The aim of this work is to use digital image processing techniques for automation of pre-operative surgery planning for selection of correct sized implantable acetabulam for Total Hip Replacement. Radiographs are not standardized in real world units and the magnification is also not uniforms. Magnification adjustment depends on the size of a patient among large to small; it is magnified for thin sized patients and reduced for large sized patients. An orthopedic surgeon must estimate the degree of magnification or reduction to select an implant of correct size (Conn K. S et al. 2002) [2]. Calculating the differences of the size of the marker displayed on the film and the actual size of the marker, the degree of magnification or reduction is estimated and compensated while selecting prosthesis. Selecting template from a library of templates the surgeon overlay it in computer over the image. Therefore, the surgeon performs the necessary measurements for templating and preoperative planning in a digital environment which is fast and precise. Till now preoperative planning was manual, landmark based template matching methods which are time consuming requiring much patience and skills of a physician. Some softwares are there for this purpose but all these end with manual detection of the position of acetabulam or related parts of hip-joints. In this paper the authors described how the detection of hip joints related areas can be automated by the image processing and calculation of the actual size, position and elongation of acetabulam can be performed in the software after detection. For x-ray imaging the brightness or darkness of the whole image varies from slide to slide and for patient to patient. All these facts are to be considered while developing the software to make standard and stable algorithm that can always detect the desired portions from the different slides. To overcome these difficulties adaptive thresholding is carried out after some preprocessing. Optimum image enhancements have been achieved using a combination of image processing steps to detect a typical organ. In this paper we will discuss imaging techniques and the choice of the templates required for preoperative planning.

\section{SCOPE AND OBJECTIVES}

The main objective of this work is to develop a technique which can automatically recognize the size of patient's acetabular implant in total hip replacement surgery. Particularly, to achieve this, objectives identified are:

i. Studying and designing techniques for the recognition of hip replacement acetabular implant size.

ii. Implementing the techniques by the software which is developed using image processing.

\section{RESEARCH BACKGROUND}

An acetabular implant size and orientation recognition technique to be developed involves several steps to be followed carefully to recognize the size of the acetabular implant accurately. In this section, the actual technique and other related matters are discussed.

\subsection{Anatomy and Morphology of A Hip Joint}

The acetabulofemoral joint, (art. coxae), is the joint between the femur and acetabulum of the pelvis, and its primary function is to support the weight of the body in both static (e.g. standing) and dynamic (e.g. walking or running) postures. The hip joint (Figure. 1) is a synovial joint formed by the articulation of the rounded head of the femur and the cup-like acetabulum of the pelvis. It is a special type of spheroidal, or ball and socket, joint where the roughly spherical femoral head is largely contained within the acetabulam and has an average radius of curvature of $2.5 \mathrm{~cm}$ (Schiffers N. et al. 2000)[7].

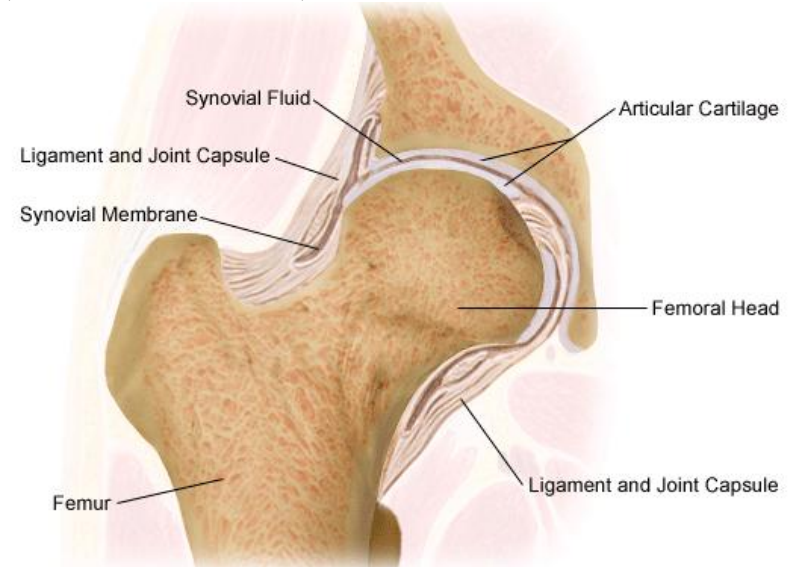

Figure1: Right hip joint - cross-section view 
The hip muscles act on three mutually perpendicular main axes, all of which pass through the center of the femoral head, resulting in three degrees of freedom (Figure. 2) and three pair of principal directions: Flexion and extension around a transverse axis (left-right); lateral rotation and medial rotation around a longitudinal axis (along the thigh); and adduction around a sagittal axis (forward-backward); and a combination of these movements (i.e. circumduction, a compound movement in which the leg describes the surface of an irregular cone)(Platzer W. 2004)[5].

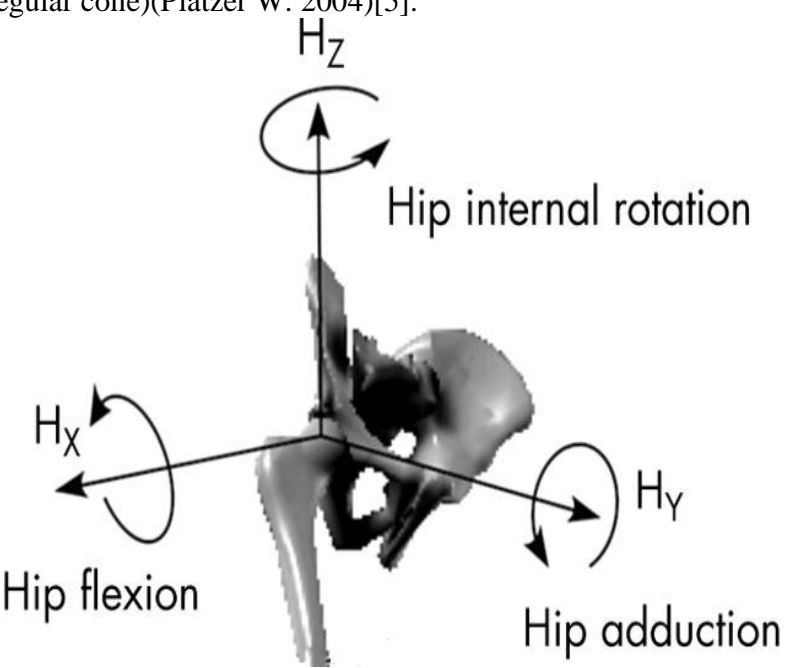

Figure2: Three degrees of hip joint freedom

The most important morphological specifications (Figure.3) which can be measured on an anteroposterior pelvic radiograph are:

- The femoral neck angle (the caput-collum-diaphyseal angle, the CCD angle) - between the longitudinal axes of the femoral neck and shaft, which normally measures approximately $126^{\circ}$ in adults,

- The acetabular inclination (the transverse angle of the acetabular inlet plane) - the angle between a line passing from the superior to the inferior acetabular rim and the horizontal plane, which normally measures $40^{\circ}$ in adults. (Schuenke M. et al. 2006)[8].
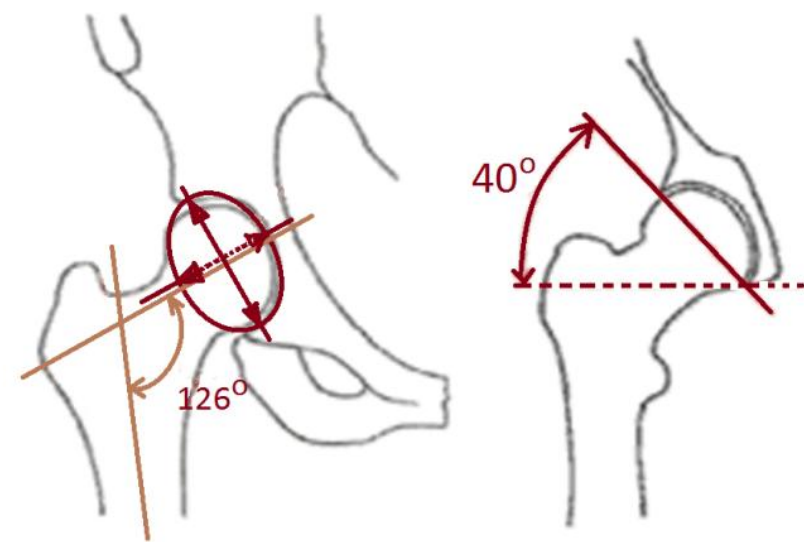

Figure3: Femoral neck angle and acetabular elongation and inclination

The next important morphological specification is the femoral neck anteversion, which is practically unmeasured in AP projection and which can be measured well by CT or MR (3D measurement methods). A perfect AP radiograph of the femur needs to account for the anteversion of the femoral neck.
Patients are required to rotate the leg internally by a mean of $15^{\circ}$. Restricted rotation of the hip in osteoarthritis sometimes makes it difficult to achieve this position. A study of the radiological dimensions of the femoral canal shows that the AP width of the medullary canal at the isthmus does not change significantly from $20^{\circ}$ internal to $40^{\circ}$ external rotation. At $20 \mathrm{~mm}$ below the lesser trochanter there is no significant change on internal rotation and an apparent increase of 1.1 $\mathrm{mm}$ with $20^{\circ}$ external rotation (Conn K. S et al. August 2001, Eckrich S. G. J et al. 1994)[2][3].

\subsection{Total Hip Prosthesis}

Total Hip replacement is a surgical procedure in which the hip joint is replaced by a prosthetic implant. Replacement of the hip joint consists of replacing both the acetabular and the femoral components as shown in Figure. 4. In orthopedic surgery generally THR is conducted in order to relieve arthritis pain or to fix severe physical joint damage as part of hip fracture treatment.

According to Monika Michalíkova et al. 2010)[4] loose sitting of total hip prosthesis is painful, and such a loose total hip is also stiff. There are two methods for securing the fixation of a total hip prosthesis to the skeleton:

i. The cemented total hip - the surgeon uses bone cement for fixation of the prosthesis to the skeleton;

ii. The cementless total hip - the surgeon impacts the total hip directly into the bed prepared in the skeleton.

With the increasing utilization of uncemented implants, templating has become more critical. With a higher risk of intra-operative fracture during insertion, it is re-assuring for the surgeon when the pre-operative prediction matches the intraoperative choice of implant. A tight interference fit is desirable when introducing the femoral component of an uncemented hip replacement. A stem which is too small may not be stable, and attempt to insert one which is too large increases the risk of intra-operative fracture. Such a complication has been reported in $3 \%$ to $24 \%$ of patients. 


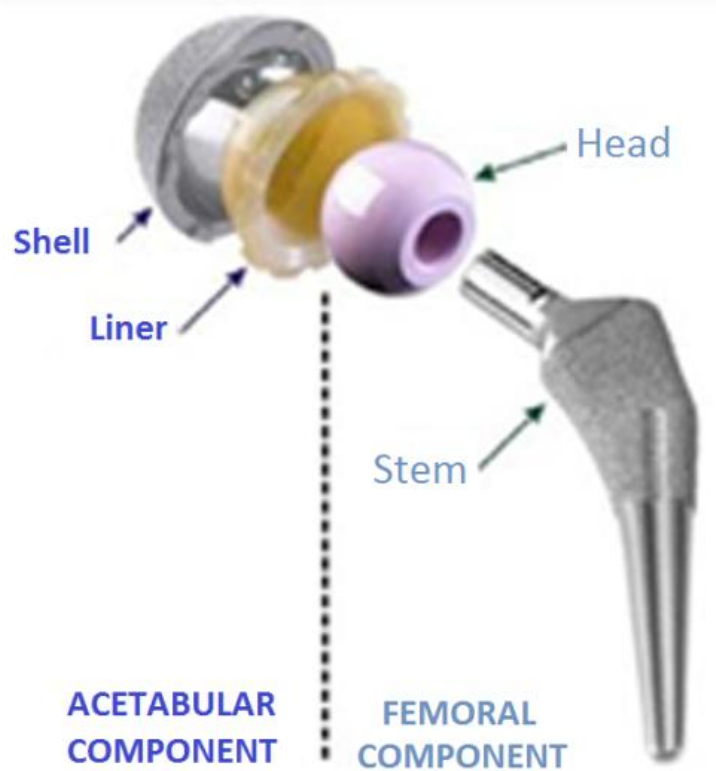

Figure4: The modular structure of total hip
prosthesis

Successful surgery requires the precise placement of implants in order that the function of the joint is optimized both biomechanically and biologically. Preoperative planning is helpful in achieving a successful result in total joint replacement. Pre-operative templating in total hip replacement helps familiarize the surgeon with the bone anatomy prior to surgery, reducing surgical time as well as complications.

This activity takes time and also is subject to mathematical error. Digital pre-operative planning allows for an image to be displayed electronically and with the aid of a known sized marker, automatically calculates the magnification and recalibrates the image so that it is sized at $100 \%$ from the perspective of the user. Typically acetate overlays and radiographs are used to determine appropriate implant size. Pre-operative planning is realized with caliper, protractor, plastic templates and $\mathrm{x}$-ray images. The measurement is time consuming and can involve multiple errors. Digital images replace radiographs, which can no longer be lost or misplaced. $\mathrm{X}$-ray images are viewed on a diagnostic grade monitor, rendering prosthetic overlays useless.

\section{MATERIALS AND METHODS}

The analysis technique aims to identify similar techniques or that has been implemented or is being implemented by other researchers around the world. An improved methodology is to be adopted using latest techniques. The proposed method has the advantage of automated recognition and calculation of the digital acetabular implant size in a digital environment

\subsection{Labview Based Automated Algorithm}

The LabView imaging tool is well known image processing software that can be used to make automated software to solve the various practical problems. In this paper 'Imaq' based algorithms are developed.

\subsection{Digital X-Ray Images}

$\mathrm{X}$-ray is a type of radiation used in medical images for diagnosing diseases like cancer and fractures. The radiologist takes X-ray images by putting on an X-ray to an opposite source of the part that needs to be imaged or convert it into films. Then, the image can be generated into films or stored digitally. The difference between a digital X-ray and a general $\mathrm{X}$-ray is that the output given in the first case is in a digital form while the general X-ray is in films. A digital X-ray may be edited and stored in a computer database. While a general $\mathrm{x}$-ray only provides a negative film output as a reference. An example of a digital X-ray of hip joint can be seen in Figure 5. Each digital $\mathrm{x}$-ray image has a different resolution depending on the amount of image compression. The purpose compression is done is to reduce the file size so that the use of memory space can be reduced, and to accelerate the transfer of files. The disadvantage of compression is that it can affect the image quality. Therefore, techniques in scaling digital XRay images with an appropriate resolution should be developed because the size of the implant identification depends on the accuracy of the image size of the patient's bone.

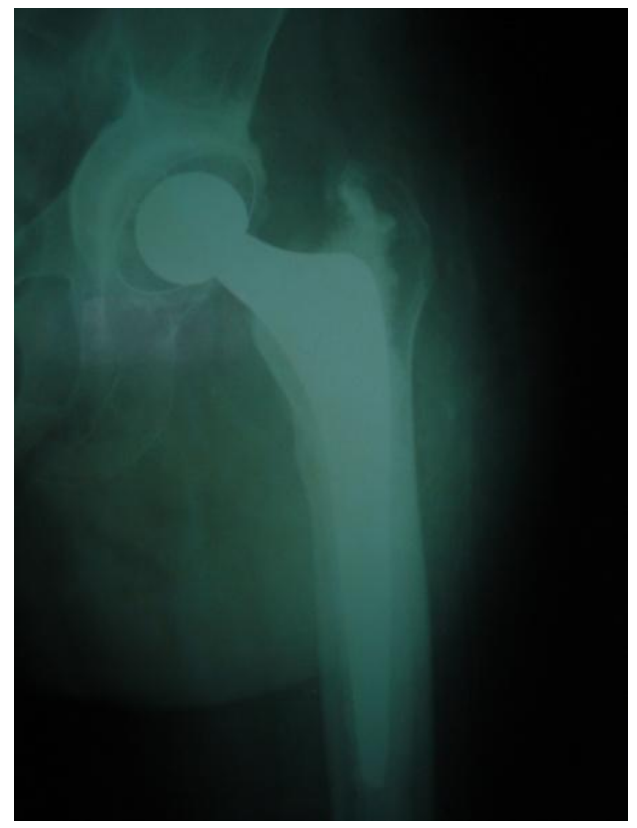

Figure5: Digital image of a hip joint

\subsection{Image and Object Recognition}

In the X-ray image the acetabular labrum is clearly identified by the physician because of its regular shape and clearly visible appearance. Acetabular labrum is a ring of cartilage that surrounds the acetabulum (the socket of the hip joint). The object is particle based, and the pixels of the particles can form a polygon area. The polygon area can be identified by testing the position of the center of mass of the particle on the digital X-ray images area. Object recognition was done by using the particle analysis on the X-ray images which lead to determine the optimum acetabular implant size.

\section{IMAGE PROCESSING TECHNIQUES}

Through the literature survey, most of the implant detection techniques use a trial and error method (W.J. Murzic et al. 2005)[12].The proposed technique has the ability to detect the size of the acetabular implant automatically. By using the basic concept of geometry, considering the distance between two points, acetabular implants were selected based on the 
patient's acetabulum diameter size from the ellipse max diameter that is extracted after processing of image using a geometrical shape reconstruction algorithm. Figure 6 shows the proposed algorithm for the acetabular implant size recognition technique.

Digital image of X-ray image is acquired either directly interfacing the computer to $\mathrm{X}$-Ray set up or by scanning the $\mathrm{X}$-ray films by a scanner. There can be only one exposure on one slide for a patient or more than one exposure from different angles. In case we have to process more than one image in the same algorithm thresholding of the X-ray slides is normalized. The image is in RGB format. We may change it in HSV model to get better gray level values in one of the grey level parameters. Here we need not use all the parameters of RGB or HSV grey levels, because the x-ray image is basically a grayscale image and we are to extract a polygonal particle (acetabular labrum) which can be significantly visible in the whole image. It was observed that if we use Value level in HSV model different slides with variable contrast and brightness were within acceptable ranges and resulted output in acceptable accuracy.The RGB-HSV conversions were given by Travis (1991)[11].

To get more accuracy we use adaptive thresholding to get closer grey level values in different images. In this method we have used Niblack's thresholding method as adaptive threasholding in segmentation algorithm. Niblack suggests calculating a threshold surface by shifting a window across the image, and use local mean and standard deviation for each center pixel in the window (Sue Wu and Adnan Amin, ICDAR 2003)[9].

The idea of adaptive thresholding is given by Rafael C. Gonzalez and Rechard E. Woods, 2007[6]. The histogram of two images may be considered an estimate of their probability density function (PDF) $p(z)$. This overall density function is the sum or mixture of two densities. Assume that the larger of the two PDFs corresponds to the background levels while the smaller one describes the gray levels of objects in the first image. The mixture probability density function describing the overall gray-lever variation in the first image - is

$$
\mathrm{p}_{\text {first }}(\mathrm{z})=\mathrm{P}_{1} \mathrm{p}_{1}(\mathrm{z})+\mathrm{P}_{2} \mathrm{p}_{2}(\mathrm{z})
$$

The mixture probability density function describing the overall gray-lever variation in the second image -is

$$
\mathrm{p}_{\text {second }}(\mathrm{z})=\mathrm{P}_{3} \mathrm{p}_{1}(\mathrm{z})+\mathrm{P}_{4} \mathrm{p}_{2}(\mathrm{z})
$$

Here, $\mathrm{P}_{1}$ and $\mathrm{P}_{2}$ are the probabilities of occurrence of the two classes of pixels; that is, $\mathrm{P} 1$ is the probability (a number) that a random pixel with value $z$ is an object pixel. Similarly, $P 2$ is the probability that the pixel is a background pixel. We are assuming that any given pixel belongs either to an object or to the background,

so that $\mathrm{P}_{1}+\mathrm{P}_{2}=1$ and

$\mathrm{P}_{3}+\mathrm{P}_{4}=1$

Both images are segmented by classifying as background all pixels with gray levels greater than a threshold $T$.

The overall probability of error is

$\mathrm{E}_{\text {first }}(\mathrm{T})=\mathrm{P}_{2} \mathrm{E}_{1}(\mathrm{~T})+\mathrm{P}_{1} \mathrm{E}_{2}(\mathrm{~T})$ and
$\mathrm{E}_{\text {second }}(\mathrm{T})=\mathrm{P}_{4} \mathrm{E}_{1}(\mathrm{~T})+\mathrm{P}_{3} \mathrm{E}_{2}(\mathrm{~T})$

Note how the quantities E1 and E2 are weighted (given importance) by the probability of occurrence of an object or background pixels. Note also that the subscripts are opposites. This is simple to explain. Consider, for example, the extreme case in which background points are known never to occur. In this case $P 2=0$.

The contribution to the overall error $(E)$ of classifying a background point as an object point $\left(E_{l}\right)$ should be zeroed out because background points are known never to occur. This is accomplished by multiplying $E_{l}$ by $P 2=0$. If background and object points are equally likely to occur, then the weights are $P_{1}=P_{2}=0.5$.

To find the threshold value for which this error is minimal requires differentiating $E(T)$ with respect to $T$ (using Leibniz's rule) and equating the result to 0 , the result is

$$
\begin{aligned}
& P_{1} p_{1}(z)=P_{2} p_{2}(z) \text { and } \\
& P_{3} p_{1}(z)=P_{4} p_{2}(z) \ldots
\end{aligned}
$$

This equation is solved for $T$ to find the optimum threshold. Note that if $\mathrm{P}_{1}=\mathrm{P} 2$,

And $\mathrm{P}_{3}=\mathrm{P}_{4}$ then the optimum threshold is where the curves for $\mathrm{p}_{1}(\mathrm{z})$ and $\mathrm{p}_{2}(z)$ intersect.

Now if $\mathrm{P}_{2}=\mathrm{P}_{4}$ then the objects in different images can be in the same level to identify. This basic adaptive thresholding method with Niblack's thresholding application is applied to all images those to be processed.

After this processing the images are segmented with background to foreground objects. The objects are divided in particles in different size and shapes. The interesting part in this step is that, the acetabulam shape is clearly visible, in carving, (in figure 6 image enhancement step), but the smaller particles are creating noise to get the exact shape of curves of acetabulam. The noises can be reduced either by different noise models in frequency domain image enhancement techniques, or by some filtering techniques in spatial domain. Here low pass filter is used in spatial domain with a contraharmonic filter of size $5 \mathrm{X} 5$ and $\mathrm{Q}=3.5$.

$$
\hat{f}(x, y)=\frac{\sum_{(s, t) \in s_{x, y}} g(s, t)^{Q+1}}{\sum_{(s, t) \in s_{x, y}} g(s, t)^{Q}}
$$

Where $\mathrm{g}(\mathrm{s}, \mathrm{t})$ is the restored value of the image $\hat{f}$ at any point $(\mathrm{x}, \mathrm{y}), \mathrm{S}_{\mathrm{xy}}$ represents the set of coordinates in a sub image window $\mathrm{m} \mathrm{X} \mathrm{n}$ and $\mathrm{Q}$ is called order of filter. For tracking acetabulam curve it is seen that only this curve line is not the biggest curve. Some other bigger curves also are there. These curves are filtered with bigger particle threasholding with object area size.

Image enhancement techniques are used to sharpen image features for display and analysis. Here we have used image enhance technique in spatial domain to extract the particular particle of our interest out of the background and surrounding particles.

After image segmentation and image enhancement there were very small particles and some very large particles which were not of our interest because they did not contribute in imaging of the desired part. So these were to be filtered out from the original image. In morphological processing high pass and low pass particle filtering has been used after some preliminary operations like erosion, dilation, closing etc. After 
the morphological processing it was clearly viewable as an elliptical shape with some discontinuities in the image. For reconstruction of the elliptical shape, another algorithm was used using the feature extraction of the curves of rare sides. In this case geometric shape fitting algorithm was used to search elliptical shape (Yonghong Xie and Qiang Ji 2002)[13].

The acetabulam face appears elliptical in shape in the image. The ellipse detection techniques in the images are derived by Yonghong Xie and Qiang Ji 2002[13]. Among the curves in the image the particular lines or part of that ellipse in curve form to be tracked for geometrical shape reformation of the acetabulam. For an arbitrary ellipse, there are five unknown parameters, $\left(\mathrm{x}_{0}, \mathrm{y}_{0}\right)$ for the center, $\alpha$ for the orientation, $(\mathrm{a}, \mathrm{b})$ for the major and minor axes. Usually we need a set of 5 edge pixels to calculate all parameters. If we use additional information at each edge pixel and/or choose special pixels, we require fewer pixels to determine the position of an ellipse. After reconstructing the geometrical shapes it overlaid the pattern and calculated the major and minor axis of the ellipse which provided us the information about the diameter size of the acetabulam and the elongation from the calculation of major and minor axis. The reconstructed ellipse is overlaid by different color and used farther calibration, analysis of dimension in terms of $\mathrm{mm}$ in real world after calibration techniques and template matching.

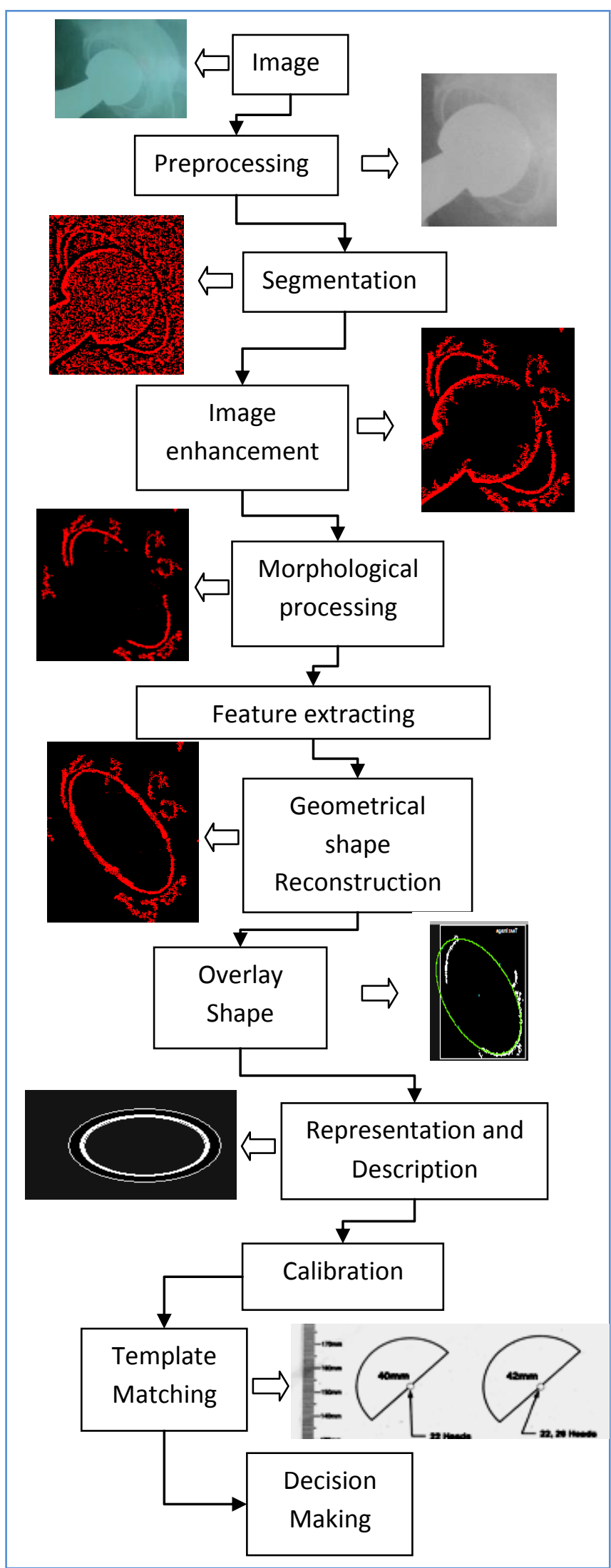

Figure 6: Block Diagram of imaging techniques used 


\section{CALIBRATION}

As explained earlier magnification is adjusted while capturing $\mathrm{X}$-Ray images each time patient to patient depending upon the size of the subject, a scale is placed on the body part to guess the actual size of the part of interest. When the major axis and minor axis of the ellipse are successfully measured according to image pixel dimension we have to calculate its actual dimensions with real world units, it is described as calibration. By calculating the difference between the size of the marker displayed on the film and the actual size of the marker, the degree of magnification or reduction was conformed.

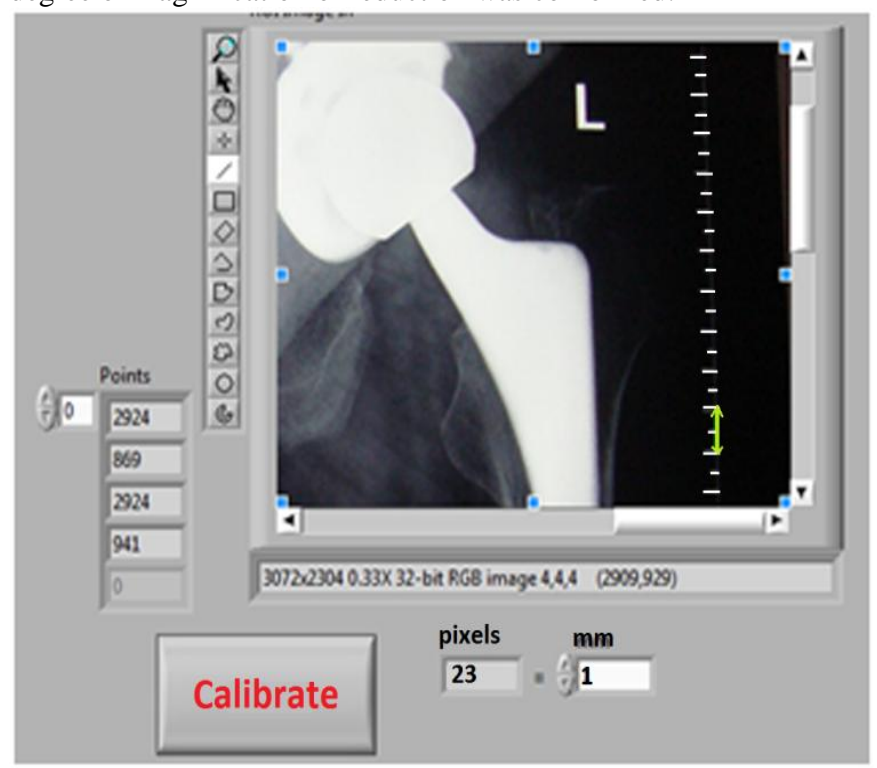

Figure 7: calibration of the digital x-ray image

In digital image the marker is marked as a series of lines. The difference between lines can be calculated in terms of pixels. If the number of pixel in between point 1 and 2 is $\mathrm{X}$ (the number of pixel between point 1and 2 has a value of $1 \mathrm{~mm}$.) then it is defined that one pixel has $1 / \mathrm{X} \mathrm{mm}$ of length and height. From this basic concept the area of particles and most other dimensions of image processing are measured in terms of real world units from the pixel values. To calculate the distance between two points in the real world is to measure the number of pixels that makes the line between the points. If the number of pixel is $\mathrm{N}$, then the distance of the line is $\mathrm{N}^{*}$ $1 / \mathrm{X} \mathrm{mm}$ in actual. Figure7 describes the calibration process in the LabView image processing tool.

\section{CALCUlations}

As explained earlier magnification is adjusted while capturing $\mathrm{X}$-Ray images each time patient to patient depending upon the size of the subject, a scale is placed on the body part to guess the actual size of the part of interest. When the major axis and minor axis of the ellipse are successfully measured according to image pixel dimension we have to calculate its actual dimensions with real world units, it is described as calibration. By calculating the difference between the size of the marker displayed on the film and the actual size of the marker, the degree of magnification or reduction was confirmed.
In digital image the marker is marked as a series of lines. The difference between lines can be calculated in terms of pixels. If the number of pixels in between point 1 and 2 is $X$ (the number of pixels between point 1 and 2 has value of $1 \mathrm{~mm}$.) then it is defined that one pixel has $1 / \mathrm{X} \mathrm{mm}$ of length and height. From this basic concept the area of particles and most other dimensions of image processing are measured in terms of real world units from the pixel values. To calculate the distance between two points in the real world is to measure the number of pixels that makes the line between the points. If the number of pixels is $\mathrm{N}$, then the distance of the line is $\mathrm{N}^{*}$ $1 / \mathrm{X} \mathrm{mm}$ in actual. Figure 7 describes the calibration process in the LabView image processing tool.

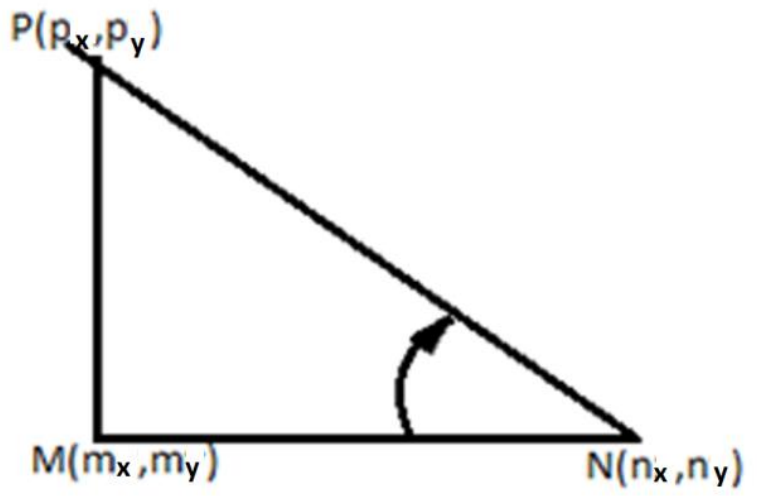

Figure 8: Angle measurement in image pixels

\section{TEMPLATE}

\subsection{Acetabular Implant Template}

Figure 9.shows the acetabular implant template used by surgeons in the THR preoperative planning procedure. In this paper the conventional templating method was used to confirm accuracy of the results. We got the dimensions directly in terms of $\mathrm{mm}$. We can choose the nearest lesser value from the available implant sizes.

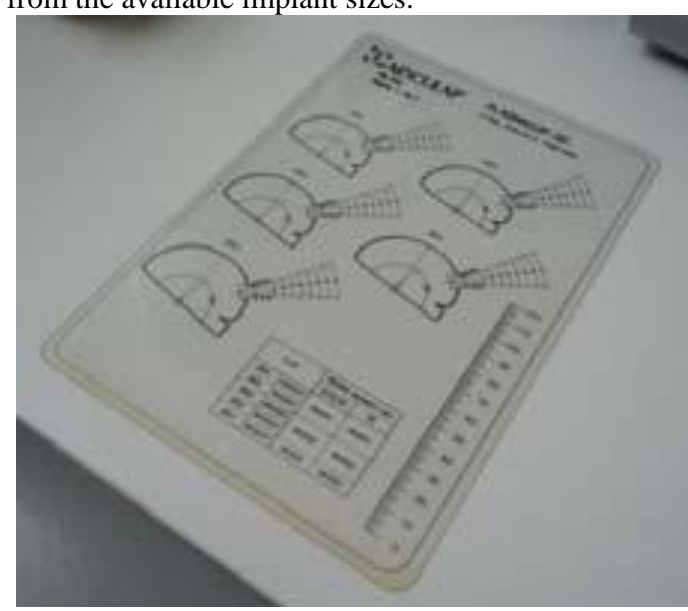

Figure 9: Acetabular implant templet

\subsection{Acetabular Implant Detection}

The size of the acetabular supplied by the PPUKM is an even number, so if there is an odd number, the even value before the odd value will be taken as the size of the acetabular implant. For example, if the distance drawn was $59.25 \mathrm{~mm}$, the value to be taken is $58 \mathrm{~mm}$. (A. Shapi et al. April 
2011)[1]. Table 1 shows the examples how to determine the acetabular size based on the distance between two points.

\section{RESULT AND DISCUSSION}

In this paper 67 images were collected of different aged patients from different sources in clinical diagnosis centers, where most of those are from PGIMER Chandigarh, India. 12 images were sampled for implementation to development of the algorithm considering as per its intensity and other quality factors. The rest of the images are used for evaluating efficiency of the algorithm. The testing images are provided in 5 classes according to diagnosis centers as the x-ray images fall approximately same intensity ranges and corresponding physicians were common. The approximate time for preoperative planning is collected from the surgeons by conversational method. And the measured sizes were collected from the diagnostic centers' data base. The images were passed through the developed algorithm with time count. The results of the comparison between manual measurements and digital measurements are shown in Table3. The corresponding size variations of all the testing images are shown in Figure 11. The other parameters i.e elongation and inclination of the acetabulam are measured in parallel. The results were shown in Table2. One of the results that shows the X-ray image, acetabular face (overlaid in green ellipse) in Front panel GUI in Labview has been shown in Figure 10. The elongation Inclination and calculated size of the acetabulam has also been shown here.

The observations from the results that the digital measurements by proposed technique follows very closely to the manual measurements in an accuracy of \pm 1 to $2 \mathrm{~mm}$ whereas the surgeons maintains the approximation of \pm 1 to 2 to choose the implant sizes as per availability.

\begin{tabular}{|c|c|c|}
\hline No. & Distance $(\mathrm{mm})$ & Acetabular size $(\mathrm{mm})$ \\
\hline 1 & 48.58 & 48 \\
\hline 2 & 57.45 & 56 \\
\hline 3 & 58.15 & 58 \\
\hline 4 & 53.36 & 52 \\
\hline 5 & 66.45 & 66 \\
\hline 6 & 69.13 & 68 \\
\hline 7 & 72.78 & 72 \\
\hline 8 & 77.67 & 76 \\
\hline
\end{tabular}

Table 1: Probable acetabular size

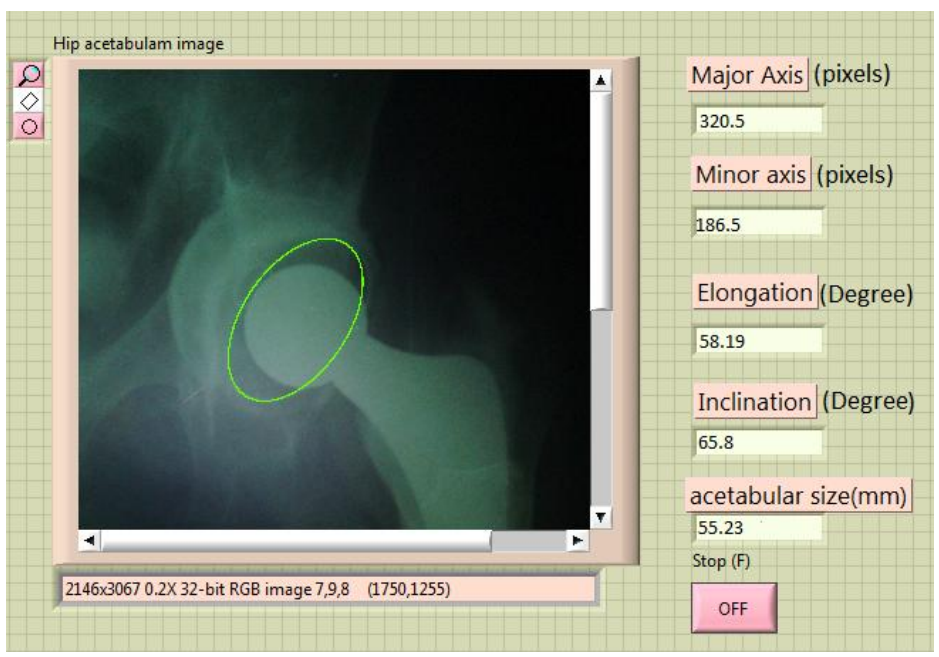

Figure 10: Final GUI for calculation of acetabulam size, elongation and inclination

\begin{tabular}{|c|c|c|c|c|}
\hline No. & Distance $(\mathbf{m m})$ & Acetabular $\operatorname{size}(\mathbf{m m})$ & Elongation( $\boldsymbol{\alpha})($ degree$)$ & Inclination $(\boldsymbol{\theta})(\mathbf{d e g r e e})$ \\
\hline 1 & 48.02 & 48 & 47.35 & 56.72 \\
\hline 2 & 55.23 & 54 & 58.19 & 65.80 \\
\hline 3 & 59.03 & 58 & 48.27 & 60.49 \\
\hline 4 & 61.45 & 60 & 50.43 & 45.44 \\
\hline
\end{tabular}

Table 2: Acetabular size ,elongations and inclinations from software

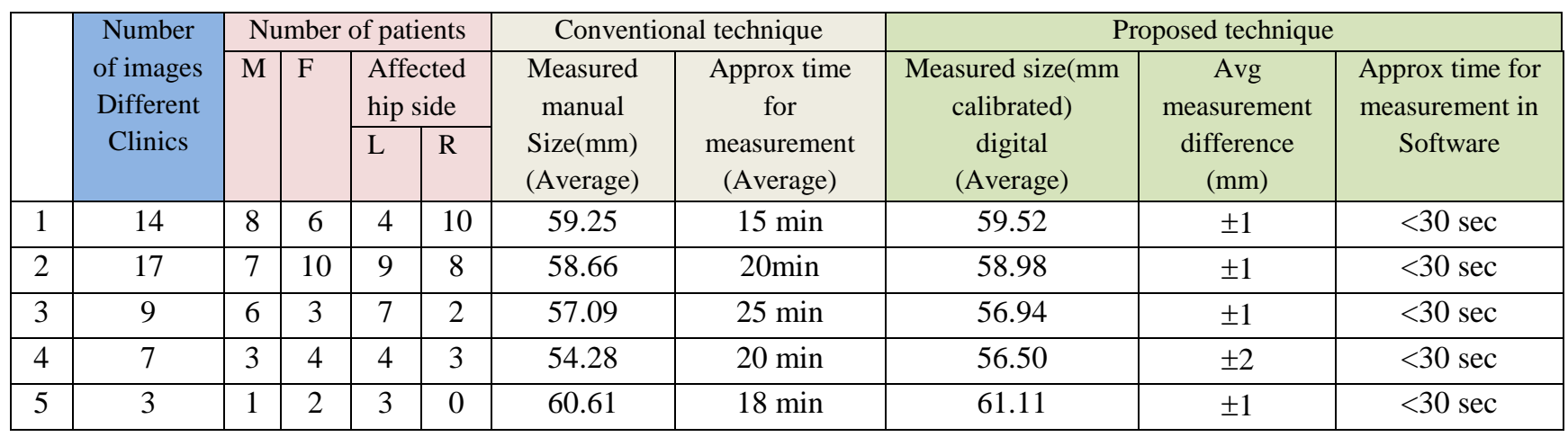

Table 3: Results on checking the algorithm on collected images and comparison of manual and digital technique 


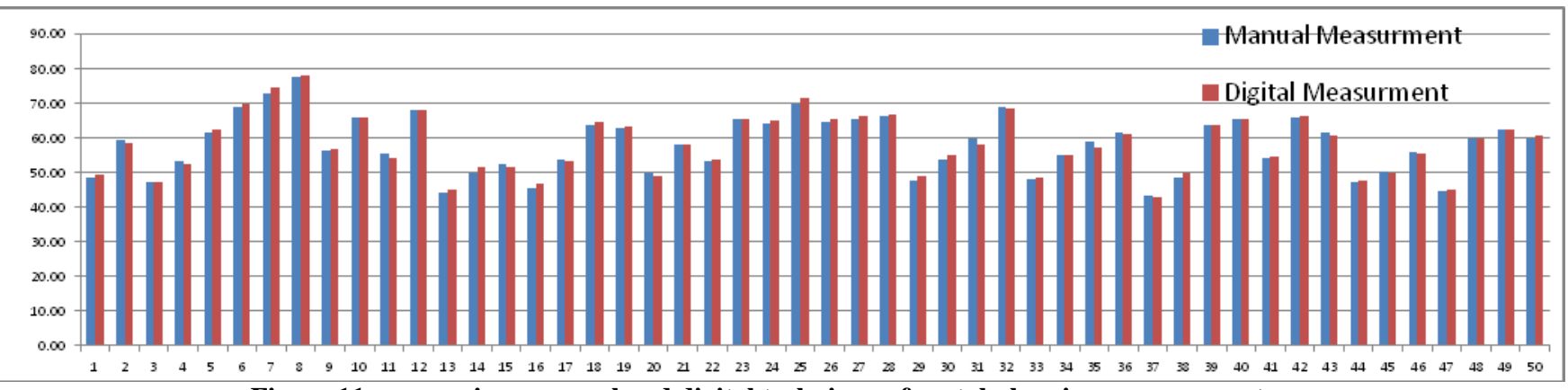

Figure 11: comparison manual and digital technique of acetabular size measurements

\section{CONCLUSION}

In surgery of THR the proposed technique has immense significance for fast and accurate calculation to choose the proper implant size. As the number of patients has been increasing the handling of operation is going to be difficult for the surgeons. This proposed technique can be helpful for a doctor as well as patient as a reliable preoperative planning tool for hemi-arthroplasty.

\section{ACKNOWLEDGMENTS}

I would like to express my deepest gratitude to Dr. Pawan Kapur, Director, CSIO, Chandigarh for providing me with an opportunity to work in the premier research organization as a Senior Research Fellow. I am also highly grateful to Dr. Vikas Bachhal Department of Orthopedics PGIMER, Chandigarh for providing me the Digital Images and contacts and direction, which helped me during my research period .I aspire to express my gratitude to Mr. Amit Laddi, Scientist 'C'DU-2 for his guidance which helped me during my research period.

\section{REFERENCES}

[1] A. Shapi, R. Sulaiman, M.K. Hasan and A.Y.M. Kassim, (IJCSIT) April 2011, "An Automated Size Recognition Technique For Acetabular Implant In Total Hip Replacement" Vol 3, No 2 :(136-249)

[2] Conn K. S.; Clarke M. T., Hallett, J. P.: 7 August 2001, J. Bone Joint Surg 2002 'A Simple Guide to Determine the Magnification of Radiographs and to Improve the Accuracy of Preoperative Templating'; 84-B:2 (69-72).

[3] Eckrich S. G. J., Noble P. C., Tullos H. S. Effect of Rotation on theRadiographic Appearance of the Femoral Canal. J. Arthroplasty 1994; (419-26)

[4] Monika Michalikova, Lucia Bednarcikova, Martin Petrík, Jozef Zivcak, Richard Rasi, Acta Polytechnica
Hungarica "The Digital Pre-Operative Planning Of Total Hip Arthroplasty” Vol. 7, No. 3, 2010.

[5] Platzer W. Color Atlas of Human Anatomy, Vol. 1, Locomotor System. 5th revised andenlarged English edition. Stuttgart, New York: Thieme; 2004 ISBN 3-13533305-1

[6] Rafael C. Gonzalez and Rechard E. Woods, Digital Image Processing, 4th Edition, 2007, ISBN 81-7758168-6

[7] Schiffers N., Schkommodau E., Portheine F., Radermacher K., Staudte H. W. "Planning and performance of orthopedic surgery with the help of individual templates". Orthopa"de. 2000 ;(636-640)

[8] Schuenke M., Schulte E., Schumacher U., Ross M. L., Lamperti D. E.:Thieme Atlas of Anatomy (2006) ISBN10: 3131420812

[9] Sue Wu, Adnan Amin "Automatic Thresholding of GrayLevel Using Multi-Stage Approach" Seventh International Conference on Document Analysis and Recognition (ICDAR 2003) IEEE

[10] TODSAPORN Fuangrod, AMNACH Khawne and MITSUHASHI Wataru "Computer-Aided Pre-Operative Planning System for Total Hip Replacement by Using 2d X-Ray Images"; SICE Annual Conference 2008

[11] Travis, D, "Effective Color Displays. Theory and Practice.”, Academic Press, 1991. ISBN 0-12-697690-2

[12] W.J. Murzic, Z. Glozman and P.Lowe. "The Accuracy of Digital (Filmless) Templating in Total Hip Replacement". 72nd Annual Meeting of the American Academic of Orthopaedic Surgeons, 2005.

[13] Yonghong Xie and Qiang Ji. "A New Efficient Ellipse Detection Method" 2002 IEEE 